\title{
Aminoglycocides as An Alternative Antibiotic Therapy Against Urinary Tract Infection in Children due to Extended-Spectrum B-Lactamase (ESBL) Producing Escherichia Coli in Carbapenem Trends
}

\author{
Muhammad Pradhika Mapindra1,2), Muhammad Pradhiki Mapindra1), \\ Muhammad Ramadhan'), Apriska Mega Sutowo Putri') \\ 1)Department of Child Health, State Hospital of Madiun, East Java \\ ${ }^{2)}$ Department of Neonatology, Soetomo Hospital, Universitas Airlangga
}

\section{ABSTRACT}

Background: Extended-spectrum $\beta$-lactamase (ESBL) is an enzyme produced by certain Escherichia coli and Klebsiella pneumoniae. ESBL can induce theantibiotic resistance for betalactam antibiotics such as penicillin, cephalosporin, etc. Frequent utilization of carbapenem may induce the risk for carbapenem resistant against ESBLs producing microorganisms. This stud aimed to communicate Aminoglycocides as an alternative antibiotic therapy against urinary tract infection in children.

Case Presentation: We present a case of 4 years childwho was admitted to the state hospital of Madiun with main complaint of 10 days high fever accompanied by phimosis appearance. Laboratory results based on urine culture show the ESBL positive Escherichia coli.

Results: We have used aminoglycoside for treating the patient. Clinical presentationin this patient start showing an improved clinical response and negative bacteriuria in the second urine test after the aminoglycoside therapy.

Conclusion: Aminoglycosides such as gentamicincan be considered to be one of the best options for treating an ESBL infection beside carbapenem in order to reduce the carbapenem trend In clinical setting of the urinary tract infection caused by ESBL Eschericia coli.

Keywords: Aminoglycosides, Carbapenem, Extended-spectrum $\beta$-lactamase, Gentamicin, urinary tract infection

\section{Correspondence:}

Muhammad Pradhika Mapindra. General Practitioner in State Hospital of Madiun, Easy Java. Email:mpradhika57@gmail.com.

\section{Cite this as:}

Mapindra MP, Mapindra MP, Ramadhan M, Putri AMS (2020). Aminoglycocides As An Alternativeantibiotic Therapy Against Childhood Urinary Tract Infection Due to Extended-Spectrum B-Lactamase Producing Escherichia Coli in Carbapenem Trends. Indones J Med. 05(03): 200-205. https://doi.org/10.26911/theijmed.2020.05.03.04.

(i) (2) Indonesian Journal of Medicine is licensed under a Creative Commons

EY AC SA Attribution-NonCommercial-ShareAlike 4.o International License.

\section{BACKGROUND}

Extended-Spectrum $\beta$-Lactamases (ESBLs) are defined as capabilities of bacterial resistance to the beta-lactam ring of antibiotics such as penicillins, cephalosporins, and aztreonamby hydrolysis of these antibiotics, and which are inhibited by $\beta$-lactamase inhibitors such as clavulanic acid. ESBL is a resistance mechanism from the specific bacteria to inactivate the antibiotic.
Some of $\beta$-lactamases derived enzymes that is well-known are TEM-1, TEM-2, and SHV-1. TEM-1 is the most common plasmid-mediated $\beta$-lactamase of ampicillin resistant enteric gram-negative bacilli (for example, Escherichia coli), while SHV1 is majorily produced by Klebsiella pneumoniae. TEM-2 is a less common member of the same group with identical biochemical properties to TEM-1. The ESBLs 
derived from TEM-1, TEM-2, or SHV-1 differ from their progenitors by as few as one amino acid (Paterson and Bonomo, 2005).

National surveys reported the presence of ESBLs Escherichia colïn 12 to 24\% isolates from Thailand, Taiwan, the Philippines, and Indonesiameanwhile 5 to $8 \%$ isolates from Korea, Japan, Malaysia and Singapore. The presence of ESBLsKlebsiella pneumoniae have been reported as low as 20 to $50 \%$ elsewhere in Asia (Paterson and Bonomo, 2005). Extended-spectrum betalactamase- (ESBL-) producing Enterobacteriaceae infections are increasingly identified with subsequent hospitalization. $E$ coli genitourinary tract infections are known as risk factor for uncomplicated cystitis, but some can ascend, affecting the kidneys and-at worst-causing overspill bacteraemia. ESBL-E coli predominantly are widespread in sewage, pets, meat, and food animals based on the definitive confirmation by whole-genome sequencing isolates. The ESBL-E coli infection in genitourinary tract reported to be the most important origin of pathogenic widespread through bloodstream lead to the sepsis and bacteremia.

Minimum inhibitory concentrations (MICs) are described as the minimum concentration of an antimicrobial that will inhibit the growth of aa bacterium or bacteria after overnight incubation. MICs are commonly used to diagnose and confirm resistance of antibiotic, but also can be used as a research tool to determine the in vitro activity of new antimicrobials (Andrews, 2001). MICs of $\beta$-lactamase-producing Enterobacteriaceae is reported as high but are still in the susceptible range. Literature determined the correlation between clinical outcomes of infection caused by ESBL microoragnisms and MICs. These studies showed that a low value of MIC is positively correlated with better clinical outcomes compared to the higher MIC (Walker, Lee and Klar, 2018). Recent literature on a MIC-based approach in settings where ESBL producers are endemic showed that cefepime and levofloxacin can both favorably achieve pharmacodynamic targets against isolates of fully susceptible Enterobacteriaceae at lowered MIC susceptibilities. There have been underlying issues with studies regarding the lack of clinical experience using these medications, as well as the conflicting reports of clinical outcomes in the literature when studying treatments for ESBL- producing Escherichia coli infection.

Carbapenem class antibiotics is admitted to be the empiric treatment of choice for ESBL-producing Enterobacteriaceae infections. Frequent carbapenem utilization in treated against ESBL producing organism which will build the carbapenem trend in clinical setting. Carbapenem trend impact to the multidrug resistance increases the risk that empirical treatment will fail, doubling the $17-18 \%$ mortality that is typical for E coli bacteraemia caused by EDBL producing E coli (Day et al., 2019). Other report also showed that carbapenem use when MIC is $\geq 4 \mathrm{mg} /$ liter contributed for the worse mortality outcomes compared to patients whose microbiological isolate MICs were lower than $4 \mathrm{mg}$.liter, demonstrating awareness and limitation of carbapenem therapy in clinical setting. The emerging incidence of community-acquired ESBL-identified infections has demonstrated a positive associationto the carbapenem resistance which canilustrate the need for alternative therapies against ESBL-producing organisms. Though prospective study demonstrating effectiveness of other therapies has been limited, there is emerging retrospective evidence in support of the use of non-carbapenem antibiotics, such ascefe- 
pime, fluoroquinolones, and piperacillin/ tazobactamin several clinical occasions (Walker, Lee, and Klar, 2018). Carbapenems with their excellent in vitro activity are reserved for children with severe clinical presentation or nosocomial infection. Carbapenem-containing combination regimens are associated with more favorable outcomes in cases with relatively low MIC $(<8 \mathrm{mg} / \mathrm{L})$ and meropenem given as a prolonged Infusion (Bitsori and Galanakis, 2019). Carbapenem trend prevention should be done by the application of noncarbapenem antibiotics, such as fourthgeneration cephalosporin which was considered as most considerable alternative to eradicate ESBL-producing organisms in in the clinical setting.

Gentamicin is an aminoglycoside antibiotic that is considered for children with an upper UTI. It is used intravenously and negligible in oral use. Gentamicin is a bactericidal antibioticthat works by binding to the bacterial ribosomes which will affect the protein synthesis. It is effective against all aerobic gram-negative rods including Pseudomonas and Proteus, and is also effective against staphylococci. Gentamicin is eliminated by glomerular filtration. The dose of gentamicin is weight related and should be calculated on the child ideal weight to avoid major adverse reactions such as ototoxicity and nephrotoxicity. Gentamicin can damage to the sensory cells of the ear and lead to hearing loss, balance problems, and tinnitus. Nephrotoxicity effect of gentamicin is occurred by thecells damage in the proximal tubule, which causes acute tubular necrosis and subsequent kidney injury. Renal function should be measured regularly, and if there is renal impairment, the interval between doses should be increased or the dose should be decreased. It is contraindicated in children with myasthenia gravis (Ramlakhan et al., 2014).

This case report will show the clinical and microbiological responses to aminoglycoside antibiotic, gentamicin in treating the febrile UTI caused by ESBL Escherichia coli in order to find the alternative antibiotic therapy and emphasize previous studies on avoiding carbapenem trends era.

\section{CASE REPORT}

A 4-year-old child presented with a ten days historyof fever and frequent urination. The urination complaint was also followed by dysuria. The other complaints are nausea and loss of appetite. Patient demonstrated axillary temperature at 38.5 degreecelsius. In addition to that, the other vital signs remain stable. Antromorphic measurement was examined during the first arrival at hospital to estimate drug doses and body fluid requirement purpose. From physical examination, patient demonstrated suprapubic pain meanwhile palpable mass in suprapubic areawas not found. On the further flank examination, tenderness on costovertebral angle was not appeared. Upon further examination in genital area, the patient presented with a sign of phymosis with unabilityto pull back patient preputium. Patient nutritional status is categorized as normal based on the analysis using water low criteria (90\%) and the CDC curve plot (body height $=103 \mathrm{~cm}$, weight $=$ $14.5 \mathrm{~kg}$, ideal body weight $=16 \mathrm{~kg}$ ).

We already ensured that the patient family consent had completely agreed to give the information related to their relative and let their relative identity to be hidden in publication.

Blood test was performed to rule out the aetiology of fever. The laboratory finding showed the blood cells remain in normal range (hemoglobin $13.2 \mathrm{~g} / \mathrm{dL}$, leukocytes 23,390/mm3, platelets 299,000 
/mm3). Urine analysisresult showed an elevated white blood cells (4-5 cell), negative erythrocytes (o-1 cell), ketonuria $(+3)$, bacteriuria $(+)$, and a slight decreasement of urine acidity ( $\mathrm{Ph}$ 6.0). Based on the previous clinical findings and laboratory results, the patient was diagnosed as febrile urinary tract infection. $400 \mathrm{mg}$ ampicillin was administered per 6 hours intravenous as an antibiotic therapy for 3 days. The patient fluid requirement was supplied by intravena fluid using D5 1/4 NS 18 drop per minute based on the daily patient requirement (1225 cc/24 hours), another supportive treatment was also considered such as the use of paracetamol $500 \mathrm{mg}$ in febrile condition (axillar temperature $\geq 385^{\circ} \mathrm{C}$ ) and domperidone for treating the vomiting and nausea. After 3 days ampiciline therapy, the patient had not shown any clinical response.

Urine culture was performed in next day after the ampiciline therapy was done to determine the suspect pathogen and sensitive antibiotic. The result of urine culture showed that ESBL phenotype was detected in the selected $E$. coli which became the responsible pathogen.

\section{RESULTS}

Gentamicin was selected as the second antibiotic therapy replacing ampicillin in treating the patient. Gentamicin was intravenously administered per 12 hours as 35mg doses for 7 days. After the administration of antibiotic and supportive treatment in 7 days, the patient condition had shown an improved clinical responseand dis not appear a febrile condition in more than 24 hours. Subsequent urine analysis revealed the condition of negative bacteriuria and decreased urine leucocytes (1-2 cell). Patient was discharged from hospital once the gentamicin therapy has completed in 7 days followed by an arrangement for circumcision procedure in next three days to minimize the risk of recurrent urinary tract infection in the future.

\section{DISCUSSION}

The patient in this case was diagnosed as febrile urinary tract infection. Urinary tract infection (UTI) is a condition of significant growth of bacteria in the urine accompanying the symptomps..After a single UTI, 15$41 \%$ of children are in risk to develop longterm complications of kidney disease, proteinuria, hypertension, and renal dysfunction in later life (Ramlakhan et al, 2014). Ampicilin was selected as the primary antibiotic therapy for treating the UTI in this case. Persistence of the febrile condition after 3 days therapystrengthen a suspicious of inadequate antibiotic therapy. Urine culture was performed to detect the ESBL producing $E$. coli and the sensitive antibiotic. Studies reported that possibilities of increased bacterial resistance to ampicillin is related to widespread use for childhood infections because it has a low side-effect profile (Ramlakhan et al., 2014).

Presence of ESBL producing organism in an infection disease results in a high rate of inadequate antibiotic therapy, hence the patients with ESBL-producing organisms needs to receive adequate antibiotic therapy immediately in 24 hours after the blood culture result revealed. Immuno-incompetent patients will be in risk for the negative impact of delayed antibiotic therapy (Puvabanditsin et al., 2019). In this case, the ESBL producing $E$. coli was resistant to Cephazoline, Ceftazidime, Ceftriaxone, Aztreonam, Ciprofloxacin, and TrimetophrimSulfametoxazhole. However, it was sensitive to gentamicin, nitrofurantoin, amikacin, meropenem, and ertapenem. Some studies reported that aminoglycoside antibiotic susceptibility such as gentamicin and 
amikacin is still high against the ESBL producing E. coli (Kumar et al., 2014).

Zohar et al. (2020) study compared aminoglycoside and carbapenemor piperacillin/ tazobactam declared that aminoglycoside is more superior in reducing the 30 day mortality rate $(13 \%)$ than carbapenem or piperacillin/tazobactam (21.2\%), although it is still inferior in reducing the recurrence of bacteriuria within 90 days and acute kidney injury in bloodstream infection caused by ESBL-producing Enterobacteriaceae (Zohar et al., 2020). Gentamicin was selected to be the antibiotic therapy in this case combined with ampicillin. Antimicrobial susceptibility in this case showed that gentamicin was the antibiotic with the lowest MIC compared to the other sensitive antibiotics, such as amikacin and nitrofurantoin. Another study of the antimicrobial susceptibility profile revealed that gentamicin has a quite higher sensitivity than nitrofurantoin (Kumar et al., 2014). The combination of ampicillin and gentamicin becomes the empiric antibiotics for childhood UTI. The amino group sidechain of ampicillininduce the penetration in outer membrane of some gram-negative bacteria accounting for its broader spectrum of activity. Ampicillin will inhibits cell-wall synthesis by the binding mechanism of the antibiotic to penicillin-binding proteins in the bacterial cell wall.

Patient in this case will be arranged for a circumcisionin the outpatient setting of which purpose is to reduce the risk of another UTI in the future. Circumcision procedureduring childhood is practiced in $>80 \%$ in Islamic countries, South Korea, and the Philippines, but theprocedure is not commonly adapted in the majority of the other Asian countries, including China, India, Japan, and Taiwan. In the countries where circumcision is commonly performed, also considered the procedure as a choice of treatment for male infants with urinary tract infection (UTI). Hence, the setting of appropriate age for circumcision remain as a question (Kanematsu, 2016). Colonization of bacteria in the inner prepuce can be modified by circumcision procedure which will reduce the potential source of bacteria causing UTI (SinghGrewal et al., 2005). Circumcision effect on reducing the preputial colonization of bacteria has been reported in several countries. There have been several nonrandomized studies reporting a decreased rate of UTI in circumcised boys compared with non-circumcised boys (Kanematsu, 2016).

Many reports and studies have shown the effectiveness of aminoglycoside antibiotics, such as gentamicin and amikacin in treating UTI patient. Even though aminoglycoside is not more superior than carbapenem as antibiotic therapy, it still show the good efficacy as an alternative antibiotic treatment in carbapenem trends. Based on our report, we recommend aminoglycoside, such as gentamicin and amikacin as an alternative for treating patient with UTI caused by ESBL Escherichia coli.

\section{AUTHOR CONTRIBUTION}

Muhammad Pradhika designed the study, analyzed data, and drafted the manuscript. Muhammad Ramadhan collected clinical data and wrote the paper. Muhammad Pradhiki collected sequence data and revised the manuscript. Mega Apriska Sutowo Putri interpreted data and substantially revised the manuscript. All authors read and approved the final version of the manuscript.

\section{CONSENT FOR PUBLICATION}

The parents of the patient provided written informed consents to publish their child's personal or clinical details along with any 
identifying images. A copy of written consent is available for review by editor-inchief of this journal.

\section{CONFLICT OF INTEREST}

The authors declare that they have no competing interests.

\section{FUNDING AND SPONSORSHIP}

This study conducted using the medical record data from the hospital. Hence, this study was low not funded by any funders.

REFERENCE
Andrews JM (2001). Determination of
minimum inhibitory concentrations. J
Antimicrob, 48(S1): $5^{-16 .}$

Bitsori M, Galanakis E (2019). Treatment of Urinary Tract Infections Caused by ESBL-producing Escherichia coli or Klebsiella pneumoniae. Pediatr Infect. Dis J. 38(12): e332-e335. doi: 10.1097/INF.0000000000002487.

Day MJ, Hopkins KL, Wareham DW, Toleman MA, Elviss N, Randall L, Cleary $P$, et al. (2019). Extended-spectrum $\beta$ lactamase-producing Escherichia coli in human-derived and food chainderived samples from England, Wales, and Scotland: an epidemiological surveillance and typing study. Lancet Infect Dis. 19(12): 1325-1335. doi: 10.1016/S1473-3099(19)30273-7.

Kanematsu A (2016). Management of phimosis as a risk factor of urinary tract infection: An Asian perspective. Urol Sci. 27(4): 190-192. doi: 10.1016/j.urols.2016.08.005.

Kumar D, Singh AK, Ali MR, Chander Y (2014). Antimicrobial susceptibility profile of extended Spectrum $\beta$ Lactamase (ESBL) producing Escherichia coli from various clinical samples. Infect Dis (Auckl). 7: 1-8. doi: 10.4137/idrt.s13820.
Paterson DL, Bonomo RA (2005). Clinical update extended-spectrum beta-lactamases: A clinical update. Clin Microbiol. 18(4): 657-686. doi: 10.1128/CMR.18.4.657.

Puvabanditsin S, et al. (2019). Extendedspectrum beta-lactamase-producing Escherichia coli meningitis and cerebral abscess in a neonate: Therapeutic Challenge. Case Reports Infec Dis. 15. doi: 10.1155/2019/6874192.

Ramlakhan S, Singh V, Stone J, Ramtahal A (2014). Clinical options for the treatment of urinary tract infections in children. Clin Med Insights: Pediatrics. 8: 31-37. https://doi.org/10.4137/CMPed.S8100.

Singh-Grewal D, Macdessi J, Craig J (2005). Circumcision for the prevention of urinary tract infection in boys: A systematic review of randomised trials and observational studies. Arch Dis Child. 90(8): 853-858. doi: 10.1136/adc.2004.049353.

Walker KJ, Lee YR, Klar AR (2018). Clinical outcomes of extended-spectrum betalactamase-producing enterobacteriaceae infections with susceptibilities among Levofloxacin, Cefepime, and Carbapenems. Can J Infect Dis Med Microbiol. doi: 10.1155/2018/3747521.

Zohar I, Schwartz O, Yossepowitch O, David SSB, Maor Y (2020). Aminoglycoside versus carbapenem or piperacillin/tazobactam treatment for bloodstream infections of urinary source caused by Gram-negative ESBL-producing Enterobacteriaceae. J Antimicrob Chemother. 75(2): 458465. doi: 10.1093/jac/dkz457. 